\title{
A Nationwide 2010-2012 Analysis of U.S. Health Care Utilization in Inflammatory Bowel Diseases
}

\author{
Welmoed K. van Deen, MD, ${ }^{*}{ }^{+}$Martijn G. H. van Oijen, PhD, ${ }^{*}$ Kelly D. Myers, BS, ${ }^{*}$ Adriana Centeno, BA, ${ }^{*}$ \\ William Howard, PhD, ${ }^{\neq}$Jennifer M. Choi, MD, ${ }^{*}$ Bennett E. Roth, MD, ${ }^{*}$ Erin M. McLaughlin, BS, \\ Daniel Hollander, MD, ${ }^{*}$ Belinda Wong-Swanson, PhD, Jonathan Sack, MD, ${ }^{\S}$ Michael K. Ong, MD, PhD," \\ Christina Y. Ha, MD, ${ }^{*}$ Eric Esrailian, MD, MPH, ${ }^{*}$ and Daniel W. Hommes, MD, PhD*
}

Background: Implementation of the 2010 Affordable Care Act (ACA) calls for a collaborative effort to transform the U.S. health care system toward
patient-centered and value-based care. To identify how specialty care can be improved, we mapped current U.S. health care utilization in patients with
inflammatory bowel diseases (IBD) using a national insurance claims database.

Methods: We performed a cross-sectional study analyzing U.S. health care utilization in 964,633 patients with IBD between 2010 and 2012 using insurance claims data, including pharmacy and medical claims. Frequency of IBD-related care utilization (medication, tests, and treatments) and their charges were evaluated. Subsequently, outcomes were put into the framework of current U.S. guidelines to identify areas of improvement.

Results: A disproportionate usage of aminosalicylates in Crohn's disease (42\%), frequent corticosteroid use (46\%, with $9 \%$ long-term users), and low rates of corticosteroid-sparing drugs (thiopurines $15 \%$; methotrexate $2.7 \%$ ) were observed. Markers for inflammatory activity, such as C-reactive protein or fecal calprotectin were not commonly used ( $8.8 \%$ and $0.13 \%$, respectively). Although infrequently used (11\%), anti-TNF antibody therapy represents a major part of observed IBD charges.

Conclusions: This analysis shows 2010-2012 utilization and medication patterns of IBD health care in the United States and suggests that improvement can be obtained through enhanced guidelines adherence.

(Inflamm Bowel Dis 2014;20:1747-1753)

Key Words: inflammatory bowel diseases, health care utilization, guidelines, costs

$\mathrm{T}$ he current U.S. health care system is suffering from a variety of clinical and economic inefficiencies. ${ }^{1-3}$ Although the focus of debates on these challenges may vary, such as excessive administration, nonadherence to guidelines, overutilization of

Supplemental digital content is available for this article. Direct URL citations appear in the printed text and are provided in the HTML and PDF versions of this article on the journal's Web site (www.ibdjournal.org).

Received for publication April 25, 2014; Accepted June 10, 2014.

From the *UCLA Center for Inflammatory Bowel Diseases, Melvin and Bren Simon Digestive Diseases Center, David Geffen School of Medicine, University of California Los Angeles, Los Angeles, California; ${ }^{\dagger}$ Department of Gastroenterology and Hepatology, Leiden University Medical Center, Leiden, the Netherlands; ${ }^{\ddagger}$ QformaInc., Santa Fe, New Mexico; and ${ }^{\S}$ Departmentof Surgery, and "Divisionof General Internal Medicine and Health Services Research, David Geffen School of Medicine, University of California Los Angeles, Los Angeles, California.

W. K. van Deen discloses research support from AbbVie; J. M. Choi discloses consultancies and speaker engagements for AbbVie, Janssen, and UCB and a research grant from Merck; C. Y. Ha discloses consultancies for Shire and UCB; D. W. Hommes discloses consultancies, speaker engagements, and research support for AbbVie, Janssen, UCB, and Ferring. The remaining authors have no conflicts of interest to disclose.

Reprints: Daniel W. Hommes, MD, PhD, UCLA Center for Inflammatory Bowel Diseases, Melvin and Bren Simon Digestive Diseases Center, David Geffen School of Medicine, University of California Los Angeles; 10945 Le Conte Avenue, 2338, Los Angeles, CA 90095 (e-mail: dhommes@mednet.ucla.edu).

Copyright (C) 2014 Crohn's \& Colitis Foundation of America, Inc.

DOI 10.1097/MIB.0000000000000139

Published online 18 August 2014. resources, uncoordinated care, and broad-based preventive failures, there is an emerging consensus that the U.S. health care system as currently implemented, with a persistent disconnection between high spending levels and discernible improvements in patient outcomes, is not sustainable.

Inflammatory bowel diseases (IBD) are prototypic chronic diseases, affecting around 1.4 million adults and children in the United States. The estimated annual disease-attributable direct costs are largely driven by hospital costs and medication, especially biological therapy. ${ }^{4,5}$ Like most chronic diseases, IBD care is beset with wide practice variations, ${ }^{6}$ provider expertise differentials (primary and specialty), and a limited evidence base for basic, let alone integrated, standards of care and quality of care. ${ }^{7}$ Fragmentation and duplication of services, suboptimal follow-up, and a lack of transparency in adherence to guidelines, particularly regarding overuse and misuse of drugs, could contribute as well to the high spending in IBD care.

We conducted a 2010-2012 insurance claims analysis encompassing 964,633 patients with IBD. The primary study objective was to assess U.S. health care utilization in patients with IBD on a national level to establish a detailed understanding of current practices in IBD management. The secondary objective was to analyze charges encountered for different aspects of IBD management and assess their relative contribution to total IBD-related health care costs. 


\section{METHODS}

\section{Claims-derived Care Analysis}

We conducted a retrospective analysis of U.S. IBD pharmacy and medical claims data, between 2010 and 2012, from Source Healthcare Analytics LLC (SHA). The data represent a significant proportion of all U.S. medical and pharmacy claims enabling quantitative/qualitative assessments of IBD-related practices and costs. Only fully adjudicated claims by both payers and providers were included. Patients with IBD were identified as having $\geq 1$ medical claim with one of the $I C D-9$ codes for Crohn's disease (CD) (555.x) or ulcerative colitis (UC) (556.x) between April 2010 and March 2012. Patients with diagnosis codes for both UC and CD were excluded from the disease specific analyses. We analyzed medical claims for patient identifiers, demographics, procedure details, charge, date, and physician information. Pharmacy claims for IBD-specific drugs (Table, Supplemental Digital Content 1, http://links.lww.com/IBD/A522) were analyzed for patient identifiers, demographics, prescription details, charge, date, insurance, and physician information. A summary of the claims data capture process is shown in Figure, Supplemental Digital Content 2, http://links.lww.com/IBD/A523. Heatmaps were generated based on UC and CD pharmacy claim counts in different U.S. regions, by physician 3-digit zip codes, divided by the assumed population sizes of these regions.

Medications were categorized into 6 groups of ascending potency: (1) aminosalicylates, (2) antibiotics, (3) budesonide, (4) systemic corticosteroids, (5) immunomodulators, and (6) biologic therapy (Table, Supplemental Digital Content 1, http://links.lww. com/IBD/A522). We determined the number of unique patients using these drugs between 2010 and 2012. Biologics, in particular intravenous infliximab and intravenous natalizumab, are commonly charged as medical claims, which were therefore included as well. For each drug group, the percentage prescribed by gastroenterologists was calculated. To determine concomitant medication use, we analyzed prescription rates in 3-month timeframes. In addition, we calculated the percentage of patients using corticosteroids for more than 105 consecutive days. To quantify the volume of patients discontinuing immunomodulators or biologics, we defined stopping as not receiving a refill within 30 days after the end date of the last prescription.

For the analysis of IBD-related procedures and tests, total claim counts, unique patient counts, and charges were extracted from the medical claims data set. IBD-related procedures were defined based on a predefined set of Current Procedural Terminology (CPT) and Healthcare Common Procedure Coding System Codes summarized in Table, Supplemental Digital Content 3, http://links.lww.com/IBD/A524. The included CPT codes cover gastrointestinal surgical procedures, anesthesia, and medical procedures; laboratory, pathology, and radiological procedures; and codes for evaluation and management. In addition, we included CPT category 2 codes for IBD-specific quality measures ${ }^{8}$ and CPT category 3 codes for gastrointestinal procedures. The included Health care Common Procedure Coding System level II codes were A-codes for stoma care, B-codes for (par)enteral therapies, and $\mathrm{J}-$, C-, and S-codes for IBD-specific drugs (Table, Supplemental Digital Content 3, http://links.lww.com/IBD/A524).

\section{Charge Analysis}

Since neither costs nor reimbursement rates are publicly known, costs in this study refer to claims-related charges and were used to assess the relative contribution of different medications and procedures to total IBD-related charges. Patient identifiers, and thus information regarding diagnoses, were only available for a subset of all pharmacy claims; therefore, deidentified claims for IBD-related medications prescribed by gastroenterologists were also collected for charge estimations. For each claim, physician and insurance information, prescription details, charges, and claim month were obtained. We corrected for the subset of patients with IBD that is not managed by gastroenterologists, using the proportion of IBD medication prescribed by nongastroenterologists in the IBD patientidentified pharmacy claims data set. To assess the charges of IBD-related procedures and tests, the medical claims data set was used. For claims without a charge, the average of charges per procedure with a known charge was used.

\section{Guidelines-derived Care Analysis}

We critically appraised and summarized all available U.S. guidelines, medical position statements, and technical reviews from the American College of Gastroenterology (ACG) and the American Gastroenterological Association (AGA). Where different sets of guidelines disagreed on specific management decisions, the most conservative measure was used in our analysis. The guidelines-derived data sets were structured in a way that would enable comparison with the claims-derived data analysis.

\section{Statistical Analysis}

Our descriptive statistics consist of patient and physician demographics, medication and medical resources utilization, and charges. All outcomes were analyzed for all patients with a diagnosis of IBD, and per diagnosis specifically (UC versus CD). All statistical analyses were performed on the SHA-retrieved data sets using SAS software (version 9.2; SAS, Cary, NC).

\section{RESULTS}

\section{Claims-derived Care Analysis}

Between 2010 and 2012, a total of 964,633 patients with IBD was identified: 501,718 patients with $\mathrm{CD}(52 \%)$ and 529,788 patients with UC (55\%); $7 \%$ had a diagnosis code for both UC and $\mathrm{CD}$. The mean age of the study population was 50.8 (SD 18.1) years (CD, 48.3 [SD 18.3] yr; UC, 52.6 [SD 17.7] yr), and $44 \%$ was male ( $43 \% \mathrm{CD}$ and $45 \% \mathrm{UC})$. In the pharmacy claims data set, a total of 413,334 patients with IBD was identified who had at least 1 pharmacy claim for IBD-related medication; 39\% of 
TABLE 1. Percentage of Patients with IBD/CD/UC Using IBD Drugs Between 2010 and 2012

\begin{tabular}{lrrr}
\hline & IBD, \% & CD, \% & UC, \% \\
\hline Aminosalicylates & 53.1 & 42.1 & 62.3 \\
Antibiotics & 23.5 & 25.2 & 20.7 \\
Budesonide & 8.0 & 12.0 & 3.7 \\
Systemic corticosteroids & 46.3 & 47.0 & 44.4 \\
Long-term corticosteroids & 8.8 & 8.3 & 8.4 \\
Thiopurines & 17.5 & 21.3 & 12.3 \\
Methotrexate & 2.6 & 3.4 & 1.6 \\
Cyclosporine & 0.2 & 0.2 & 0.2 \\
Biologics & 11.0 & 16.8 & 3.5 \\
& & & \\
\hline
\end{tabular}

these claims were processed by commercial insurers, $30 \%$ by a pharmacy benefit manager, $14 \%$ by Medicare, $6 \%$ by Medicaid, $8 \%$ by an employer group, and $3 \%$ paid cash (Table, Supplemental Digital Content 4, http://links.lww.com/IBD/A525). Geographical heatmaps that show the relative amount of claims per 3-digit zip code area are provided in Figure, Supplemental Digital Content 5, http://links.lww.com/IBD/A526 for CD and UC.

\section{Pharmacy Claims Analysis}

Table 1 summarizes observed use of IBD medication, subdivided for $\mathrm{CD}$ and UC. In our study population, $62 \%$ of patients with UC and $42 \%$ of patients with CD used aminosalicylates. In total, $32 \%$ of all aminosalicylate claims were prescribed for patients with CD. Antibiotics were used by $21 \%$ of patients with UC and $25 \%$ with $\mathrm{CD}$, and corticosteroids were used in $46 \%$ of patients with IBD (CD 47\%, UC 44\%). Long-term use of corticosteroids was observed in $8.8 \%$ of patients $(19 \%$ of all corticosteroid users) within the study period. Concomitant use of corticosteroid-sparing medication, i.e., immunosuppressives, was low (15\% used thiopurines concomitant with corticosteroids, $2.7 \%$ used methotrexate) (Table 2). In total, $18 \%$ of patients used thiopurines (CD 21\%, UC 12\%), 2.6\% methotrexate, and $0.2 \%$ cyclosporine. Of patients with UC receiving thiopurines, 59\% continued the use of aminosalicylates; for methotrexate, this was $31 \%$. We observed that $54 \%$ of patients who used immunomodulators stopped, of whom $73 \%$ restarted again. The number of patients with CD who used infliximab, adalimumab, certolizumab pegol, or natalizumab was $6.0 \%, 9.2 \%, 2.5 \%$, and $0.1 \%$, respectively; for patients with UC, these rates were $2.1 \%, 1.3 \%, 0.2 \%$, and $0 \%$, respectively. Of patients taking biologics, $48 \%$ stopped, of whom $74 \%$ restarted. The majority of biologics (69\%), immunomodulators (63\%), aminosalicylates (64\%), and budesonide $(69 \%)$ were prescribed by gastroenterologists. Nongastrointestinal specialists prescribed most of the corticosteroids (70\%) and antibiotics $(71 \%)$.

\section{Medical Claims Analysis}

A total of $12,374,156$ medical claims were identified between 2010 and 2012, covering 6405 different claim codes. Of these codes 1750 (27\%) were IBD-related, corresponding with $9,818,429$ claims $(79 \%$ of the total claims). The most common claims were 15-minute office visit (684,790 claims), 25-minute office visit (641,367 claims), complete blood count $(514,459$ claims), venipuncture (513,527 claims), and colonoscopy with biopsies (467,980 claims).

The average rate of annual outpatient clinic visits was $94 \%$, ER visits $11 \%$, hospitalizations $6.5 \%$, and surgeries $2.8 \%$ (Table 3). The rate of outpatient clinic visits was higher for CD (97\%) compared with UC (74\%). Annual colonoscopy rates were $25 \%$ for CD and $34 \%$ for UC. The annual rate of imaging (ultrasound, magnetic resonance imaging, or computed tomography abdomen/pelvis) was $18 \%$, of complete blood count $32 \%$, and of liver enzyme tests $20 \%$. Annual rates of inflammatory activity assessment using biomarkers were as follows: C-reactive protein $8.8 \%$, ESR $9.7 \%$, fecal calprotectin $0.13 \%$, fecal lactoferrin $0.13 \%$, and fecal leukocytes $0.32 \%$. During the study period, $1.0 \%$ of patients underwent a dual-energy x-ray absorptiometry scan. Determination of the rate of thiopurine methyltransferase testing and thiopurine metabolites did not result in reliable results, because multiple CPT codes are used for these

TABLE 2. Concomitant Drug Use

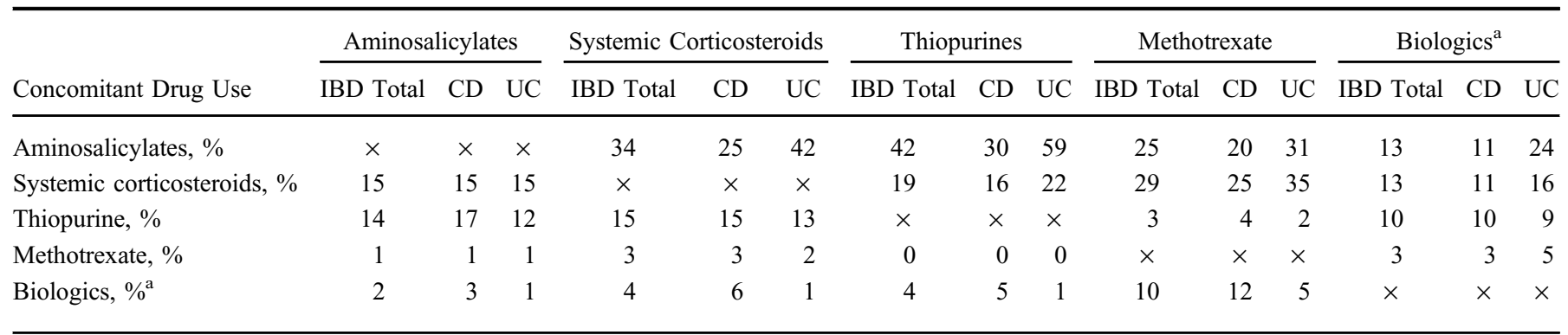

Percentages of patients on drug A (columns) concomitantly using drug B (rows).

${ }^{\mathrm{a} B e c a u s e}$ this analysis was performed using pharmacy claims and infliximab is mostly charged as a medical claim, infliximab use is underestimated in this analysis. 
TABLE 3. Observed Average Annual Rate for Hospital Visits, Endoscopies, Surgeries, Laboratory Investigations, and Imaging

\begin{tabular}{|c|c|c|c|}
\hline & $\mathrm{IBD}, \%$ & $\mathrm{CD}, \%$ & $\mathrm{UC}, \%$ \\
\hline ER visit & 10.7 & 15.1 & 4.5 \\
\hline Outpatient visit & 93.8 & 97.4 & 74.2 \\
\hline Hospitalization & 6.5 & 7.6 & 4.3 \\
\hline Endoscopy total & 42.0 & 34.1 & 44.2 \\
\hline Upper GI endoscopy & 5.8 & 6.2 & 4.7 \\
\hline Colonoscopy & 31.3 & 25.0 & 33.9 \\
\hline IBD-related surgery total & 2.8 & 3.3 & 1.6 \\
\hline Resection colon/ileocecal & 1.1 & 1.2 & 0.8 \\
\hline Fistula/abscess surgery & 0.6 & 0.9 & 0.1 \\
\hline $\mathrm{CBC}$ & 32.5 & 39.5 & 18.6 \\
\hline CRP & 8.8 & 11.2 & 4.1 \\
\hline ESR & 9.7 & 12.0 & 4.8 \\
\hline Liver enzymes & 20.4 & 24.9 & 11.4 \\
\hline Fecal calprotectin & 0.1 & 0.2 & 0.1 \\
\hline Fecal lactoferrin & 0.1 & 0.1 & 0.1 \\
\hline Fecal leukocytes & 0.3 & 0.3 & 0.3 \\
\hline Influenza vaccination $^{\mathrm{a}}$ & 1.8 & 1.9 & 1.3 \\
\hline Pneumococcal vaccination $^{a}$ & 0.5 & 0.5 & 0.4 \\
\hline Hepatitis B vaccination $^{a}$ & 0.1 & 0.2 & 0.1 \\
\hline TB screen ${ }^{a}$ & 0.8 & 1.1 & 0.4 \\
\hline Hepatitis B screening $^{\mathrm{a}}$ & 0.8 & 1.0 & 0.4 \\
\hline US/MRI/CT abdomen/pelvis & 18.1 & 22.6 & 11.3 \\
\hline DXA scan & 0.6 & 0.8 & 0.3 \\
\hline
\end{tabular}

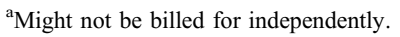

$\mathrm{CD}$, Crohn's disease; $\mathrm{CBC}$, complete blood count; CRP, C-reactive protein; CT, computed tomography; DXA, dual-energy x-ray absorptiometry; ER, emergency room; ESR, erythrocyte sedimentation rate, GI, gastrointestinal tract; MRI, magnetic resonance imaging; TB, tuberculosis; UC, ulcerative colitis, US: ultrasound.

tests and these CPT codes are also used for other tests. The annual observed rate of tuberculosis skin or quantiferon tests, recommended for screening in patients starting with biological treatment was $0.8 \%$, and of hepatitis B screening $0.8 \%$, and annual rates of influenza and pneumococcal vaccinations were $1.8 \%$ and $0.5 \%$, respectively. However, many of those might not be billed for independently.

\section{Charges}

Annual U.S. medical claim charges for patients with IBD were in total $\$ 4.6$ billion, of which $86 \%$ ( $\$ 3.9$ billion) were directly related to IBD care. The medical claim with the highest share in these charges was infliximab (35\%), followed by colonoscopy with biopsies (4.6\%) and intravenous infusion of chemotherapy/biologics (3.5\%). Furthermore, in total, $22 \%$ of the IBD-related medical claim charges were related to endoscopies and surgeries (including pathology and anesthesia charges), 13\% to physician consultation services, and $9 \%$ were for laboratory tests (Fig. 1A). Patients with a diagnosis code for CD had, on average, higher annual charges and more claims (mean annual charge of $\$ 5004$ with 6 claims on average) compared with patients with UC (mean annual charge of $\$ 2381$, with 3 claims on average). Annual IBD-related pharmacy claims were estimated to account for a total of $\$ 2.9$ billion annually. In total, $54 \%$ of those were for aminosalicylates (of which $32 \%$ for patients with CD) and $21 \%$ for biologics (Fig. 1B).

\section{Guidelines-derived Care Analysis}

We identified 7 guidelines/medical position statements published between 2003 and 2010 with recommendations relevant for IBD care; 4 by the American Gastroenterological Association $(\mathrm{AGA})^{9-12}$ (all accompanied by technical reviews ${ }^{13-16}$ ) and 3 by the American College of Gastroenterology (ACG). ${ }^{17-19}$ Four focused on IBD management, ${ }^{10,12,17,18} 2$ on colorectal cancer screening, ${ }^{9,19}$ and 1 on osteoporosis management in gastrointestinal diseases. ${ }^{11}$ None of the guidelines offered detailed recommendations on the

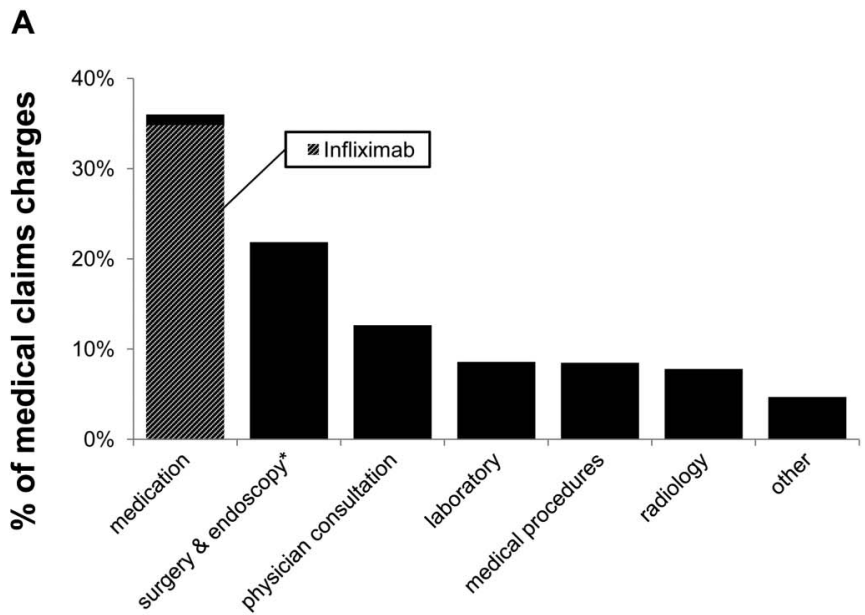

B

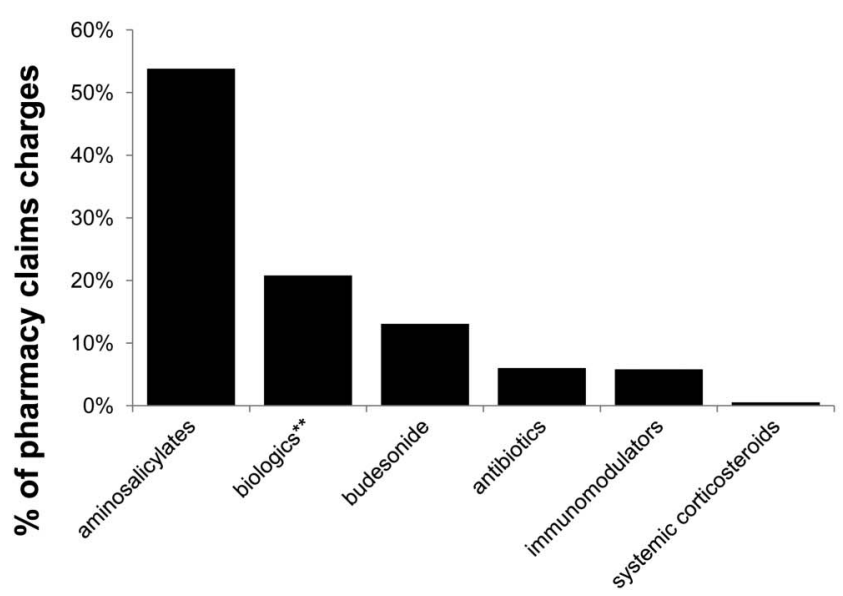

FIGURE 1. Origin of charges in medical claims data set (A) and pharmacy claims data set (B). *Includes anesthesia and pathology. ${ }^{* *}$ The majority of infliximab and natalizumab charges is charged as a medical claim and is therefore not included in this graph. 


\section{TABLE 4. Expected Medication Use According to Guidelines Compared with Observed Values}

\begin{tabular}{llll}
\hline Medication & \multicolumn{1}{c}{ Guidelines } & Expected & Observed \\
\hline Aminosalicylates & $\begin{array}{c}\text { Recommended for UC, not/minimally } \\
\text { effective for CD } \\
\text { Only recommended for pouchitis or fistula }\end{array}$ & $\begin{array}{c}\text { No aminosalicylates } \\
\text { for CD } \\
\text { Unknown }\end{array}$ & $42.1 \%$ in CD \\
$\begin{array}{l}\text { Ciprofloxacin/ } \\
\text { metronidazole }\end{array}$ & Recommended for UC not for CD & No budesonide in UC & $\begin{array}{l}3.7 \% \text { budesonide in UC } \\
\text { Budesonide }\end{array}$ \\
$\begin{array}{l}\text { Corticosteroids } \\
\begin{array}{l}\text { Immunomodulators/ } \\
\text { biologics }\end{array}\end{array}$ & $\begin{array}{l}\text { For induction of remission, no long-term use } \\
\text { Recommended for corticosteroid sparing }\end{array}$ & $\begin{array}{l}\text { No long-term use } \\
46.3 \% \text { used } \\
\text { corticosteroids }\end{array}$ & $\begin{array}{c}\text { 15\% of corticosteroid users used concomitant } \\
\text { thiopurines, } 2.7 \% \text { methotrexate. In total 11.0\% } \\
\text { biologics use }\end{array}$ \\
\hline
\end{tabular}

annual frequency of clinic visits, laboratory visits, and endoscopies, with the exception of colorectal screening protocols. Extracted care recommendations from all guidelines are summarized in Table, Supplemental Digital Content 6, http:// links.lww.com/IBD/A527.

An overview of expected rates of medication and medical resource utilization according to guidelines versus the observed rates is provided in Tables 4 and 5, respectively. Summarized, we found that although aminosalicylate treatment is not recommended in patients with $\mathrm{CD}, 42.1 \%$ of patients with $\mathrm{CD}$ were prescribed aminosalicylates during the 2-year study period, which alone accounts for at least $17 \%$ of total pharmacy charges. Metronidazole and ciprofloxacin, indicated for treatment of pouchitis in UC, active fistulizing disease in $\mathrm{CD}$, and to treat infectious complications, were prescribed to $23 \%$ of patients. However, the claims data did not allow a more detailed analysis on indications for antibiotic use.

Corticosteroid-sparing medication was used sparsely in conjunction with corticosteroid therapy $(15 \%$ thiopurines and
2.7\% methotrexate), whereas long-term corticosteroid use was observed in $9 \%$ of patients. Although $9 \%$ of patients used corticosteroids for more than 105 days consecutively, only $1 \%$ underwent a dual-energy x-ray absorptiometry scan. Furthermore, we found a low use of surrogate biomarkers for assessment of inflammation, such as C-reactive protein and/or fecal calprotectin $(8.8 \%$ and $0.13 \%$, respectively).

\section{DISCUSSION}

In this study, we report on U.S. health care utilization in patients with IBD and found unexpected discrepancies with U.S. guidelines. This was demonstrated by a disproportionate rate of aminosalicylate use in $\mathrm{CD}$, common corticosteroid use (including long-term), and a low rate of corticosteroid-sparing drugs. In addition, we found only infrequent usage of surrogate biomarkers, such as C-reactive protein and/or fecal calprotectin.

IBD-related health expenditures are among the highest in the U.S. health care system. ${ }^{20}$ A 2012 study based on patientreported expenditures from 556 patients with IBD estimated

TABLE 5. Expected Rates of Tests and Procedure in the Data Set According to Guidelines, Compared with the Observed Values

\begin{tabular}{|c|c|c|c|}
\hline Procedures & Guidelines & Expected & Observed \\
\hline Colonoscopy & $\begin{array}{l}1 \times \text { per } 1-3 \text { years, } 8 \text { years after } \\
\text { diagnosis }\end{array}$ & $\begin{array}{l}\text { Patients with } \mathrm{UC}>8 \text { yr after diagnosis: } \\
33.3 \% \text { annual colonoscopy }\end{array}$ & Patients with UC: $33.9 \%$ annual rate \\
\hline DXA scan & Patients $>3$ mo corticosteroids & $\begin{array}{l}9 \% \text { of patients } \geq 1 \text { episode of long-term } \\
\quad \text { corticosteroids }\end{array}$ & $1.0 \%$ of patients \\
\hline $\begin{array}{l}\text { Complete } \\
\text { blood } \\
\text { count }\end{array}$ & $\begin{array}{l}1 \times \text { per } 1-2 \text { weeks initially, then } 1 \times \text { per } \\
3 \text { months }\end{array}$ & $\begin{array}{l}\text { Patients on immunomodulators per } \\
\text { quarter: } 8.7 \% \text { thiopurines, } 1.1 \% \\
\text { methotrexate }\end{array}$ & $8.1 \% 3$-mo rate (32.5\% annual rate) \\
\hline $\begin{array}{l}\text { Liver } \\
\quad \text { enzymes }\end{array}$ & $\begin{array}{l}\text { Patients on thiopurines/methotrexate: } \\
\text { routinely/every } 1-2 \text { mo }\end{array}$ & $\begin{array}{l}\text { Patients on immunomodulators per } \\
\text { quarter: } 8.7 \% \text { thiopurines, } 1.1 \% \\
\text { methotrexate }\end{array}$ & $3.4 \%$ 2-mo rate (20.4\% annual rate) \\
\hline
\end{tabular}

CRP, C-reactive protein; DXA, dual-energy x-ray absorptiometry; ESR, erythrocyte sedimentation rate; UC, ulcerative colitis 
annual IBD-related costs in the United States to be $\$ 2.9$ billion, ${ }^{20}$ whereas another claims analysis of 19,420 patients with IBD estimated annual disease-attributable direct costs to be $\$ 6.3$ billion. ${ }^{21}$ Although we were not able to access actual costs in our study, we were able to assess the relative contribution of the different facets of IBD treatment to total IBD-related charges. We identified biologics to be a major cost component in IBD care, although their use was restricted to only $11 \%$ of patients with IBD in the observation period. Aminosalicylates accounted for $54 \%$ of pharmacy claim charges, while $32 \%$ of the prescriptions were prescribed for patients with $\mathrm{CD}$, which is not supported by current guidelines.

Medical insurance claims databases are increasingly used in health outcomes research, and these data present both opportunities and limitations. ${ }^{22}$ A major advantage is that claims are anonymous, plentiful, and available in electronic format. Limitations include the focus of claims on reimbursement, which is not designed for research purposes; no health outcomes or treatment goals are available, diagnoses cannot be formally confirmed, and medical utilization without insurance coverage, such as influenza vaccinations at the workplace, is not captured. Also, because only claims processed through medical clearinghouses could be captured in our data set, we were likely not able to capture all U.S. patients with IBD, a fraction of claims for the identified patients might not have been included, and no reimbursement rates were available.

An insurance claims analysis including 19,420 patients with IBD by Kappelman et $\mathrm{al}^{5}$ found much higher utilization rates because of more stringent inclusion criteria, thereby excluding patients with a mild disease phenotype, patients whom our study aimed to include. In contrast, utilization rates reported in a Northern California study analyzing 8787 patients with IBD were very similar to our observations, except for the number of outpatient visits ${ }^{23}$ (Table, Supplemental Digital Content 7, http://links.lww.com/IBD/A528). This study also reported a decline in prolonged steroid exposure from $14 \%$ in 1998-1999 to 9\% in 2004-2005 annually in CD, and interestingly, an increase in UC from $11 \%$ to $14 \%$. Infliximab use increased from $1 \%$ to $5 \%$ in $\mathrm{CD}$ and from $<0.1 \%$ to $0.4 \%$ in UC. ${ }^{23}$ Our results are in line with these findings and confirm that similar patterns are observed on a national level.

The observed discrepancies between guidelines and observed care could be explained in different ways. The New England Health Institute identified 4 major barriers to guideline adherence. $^{24}$

1. The current payment system is problematic, because we pay for volume of procedures rather than for outcomes;

2. A lack of information technology systems is a barrier because physicians often have insufficient access to guidelines at the point of care and because information technology does not yet adequately support clinical decision making;

3. The culture, beliefs, and habits of physicians could be barriers because many doctors receive little or no comparative feedback on their performance; and
4. The current process of development of guidelines presents an obstacle to adherence. In particular, the lack of transparency in guideline development leads to a lack of trust among physicians, while guidelines themselves often lack sufficient flexibility and relevance to clinical practice; many guidelines do not reflect the complexity and context in which real-world clinical decisions must be made. ${ }^{24}$

In summary, in our claims data set of 964,633 patients with IBD, unprecedented in size, we found relevant discrepancies between daily care and guideline recommendations on a national level. The guidelines themselves, in this case for a prototypic chronic disease, need to be assessed and updated to enable development of optimal care pathways that are both clinically and economically efficacious. Future research will need to show the effect of improved guidelines on adherence, quality of care, and cost-effectiveness.

\section{REFERENCES}

1. Porter ME, Lee TH. The Strategy that will Fix health care. Harv Business Rev. 2013;91:50-70.

2. Arrow K, Auerbach A, Bertko J, et al. Toward a 21st-century health care system: recommendations for health care reform. Ann Intern Med. 2009; 150:493-495.

3. Song Z, Lee TH. The era of delivery system reform begins. JAMA. 2013; 309:35-36.

4. Park KT, Bass D. Inflammatory bowel disease-attributable costs and costeffective strategies in the United States: a review. Inflamm Bowel Dis. 2011;17:1603-1609.

5. Kappelman MD, Porter CQ, Galanko JA, et al. Utilization of health care resources by U.S. children and adults with inflammatory bowel disease. Inflamm Bowel Dis. 2011;17:62-68.

6. Colletti RB, Baldassano RN, Milov DE, et al. Variation in care in pediatric Crohn disease. J Pediatr Gastroenterol Nutr. 2009;49:297-303.

7. Hommes D, Colombel JF, Emery P, et al. Changing Crohn's disease management: need for new goals and indices to prevent disability and improve quality of life. J Crohns Colitis. 2012;6(suppl 2):S224-S234.

8. The American Gastroenterological Association. Adult inflammatory bowel disease physician performance measures set, August 2011. Available at: http://www.gastro.org/practice/quality-initiatives/IBD_Measures. pdf. Accessed January 9, 2014.

9. Farraye FA, Odze RD, Eaden J, et al. AGA medical position statement on the diagnosis and management of colorectal neoplasia in inflammatory bowel disease. Gastroenterology. 2010;138:738-745.

10. Lichtenstein GR, Abreu MT, Cohen R, et al. American Gastroenterological Association Institute medical position statement on corticosteroids, immunomodulators, and infliximab in inflammatory bowel disease. Gastroenterology. 2006;130:935-939.

11. American Gastroenterological Association Medical position statement: guidelines on osteoporosis in gastrointestinal diseases. Gastroenterology. 2003;124:791-794.

12. American Gastroenterological Association Medical position statement: perianal Crohn's disease. Gastroenterology. 2003;125:1503-1507.

13. Farraye FA, Odze RD, Eaden J, et al. AGA technical review on the diagnosis and management of colorectal neoplasia in inflammatory bowel disease. Gastroenterology. 2010;138:746-774.

14. Lichtenstein GR, Abreu MT, Cohen R, et al. American Gastroenterological Association Institute technical review on corticosteroids, immunomodulators, and infliximab in inflammatory bowel disease. Gastroenterology. 2006;130:940-987.

15. Bernstein CN, Leslie WD, Leboff MS. AGA technical review on osteoporosis in gastrointestinal diseases. Gastroenterology. 2003;124:795-841.

16. Sandborn WJ, Fazio VW, Feagan BG, et al. AGA technical review on perianal Crohn's disease. Gastroenterology. 2003;125:1508-1530.

17. Lichtenstein GR, Hanauer SB, Sandborn WJ. Management of Crohn's disease in adults. Am J Gastroenterol. 2009;104:465-483. 
18. Kornbluth A, Sachar DB. Ulcerative colitis practice guidelines in adults: American College of Gastroenterology, Practice Parameters Committee. Am J Gastroenterol. 2010;105:501-523.

19. Levin B, Lieberman DA, McFarland B, et al. Screening and surveillance for the early detection of colorectal cancer and adenomatous polyps, 2008: a joint guideline from the American Cancer Society, the US Multi-Society Task Force on Colorectal Cancer, and the American College of Radiology. Gastroenterology. 2008;134: $1570-1595$.

20. Gunnarsson C, Chen J, Rizzo JA, et al. Direct health care insurer and outof-pocket expenditures of inflammatory bowel disease: evidence from a US national survey. Dig Dis Sci. 2012;57:3080-3091.
21. Kappelman MD, Rifas-Shiman SL, Porter CQ, et al. Direct health care costs of Crohn's disease and ulcerative colitis in US children and adults. Gastroenterology. 2008;135:1907-1913.

22. Ferver K, Burton B, Jesilow P. The use of claims data in health care research. Open Public Health J. 2009;2:11-24.

23. Herrinton LJ, Liu L, Fireman B, et al. Time trends in therapies and outcomes for adult inflammatory bowel disease, Northern California, 19982005. Gastroenterology. 2009;137:502-511.

24. Kenefick H, Lee J, Fleishman V. Improving physician adherence to clinical practice guidelines: barriers and strategies for change. 2008. Available at: http://www.nehi.net/writable/publication_files/file/cpg_report_final.pdf. Accessed January 9, 2014. 